\title{
Shape Coexistence in Neutron-rich Strontium Isotopes at $\mathrm{N}=60$
}

\section{E. Clément*}

GANIL, CEA/DRF-CNRS/IN2P3, F-14076 Caen Cedex 05, France

E-mail: clementeganil.fr

\section{Zielińska}

Irfu, CEA, Université Paris-Saclay, F-91191 Gif-sur-Yvette, France

\footnotetext{
The structure of neutron-rich ${ }^{96,98} \mathrm{Sr}$ nuclei was investigated by low-energy Coulomb excitation of radioactive beams at the REX-ISOLDE facility, CERN, with the MINIBALL spectrometer. A rich set of transitional and diagonal $E 2$ matrix elements has been extracted from the differential Coulomb-excitation cross sections. The results support the scenario of a shape transition at $\mathrm{N}=60$, giving rise to the coexistence of a highly deformed prolate and a spherical configuration in ${ }^{98} \mathrm{Sr}$ with low configuration mixing.
}

The 26th International Nuclear Physics Conference

11-16 September, 2016

Adelaide, Australia

${ }^{*}$ Speaker. 


\section{Introduction}

Neutron-rich $\mathrm{A} \sim 100$ nuclei are amongst the best examples of the interplay of microscopic and macroscopic effects in nuclear matter. A rapid onset of quadrupole deformation is known to occur at around $\mathrm{N}=60$, making this region an active area for both experimental and theoretical studies. Mass and charge radii measurements in this region of the nuclear chart showed that the increase of binding energy in the $\mathrm{Rb}, \mathrm{Sr}, \mathrm{Y}$ and $\mathrm{Zr}$ isotopes at $\mathrm{N}=60$ is associated to a dramatic increase of the ground-state deformation. The low- $Z$ border of this phenomenon has been recently established by means of mass measurements in the $\mathrm{Kr}$ isotopic chain, where no deviation from the standard trend toward the drip line was observed at $\mathrm{N}=60$ [1], and from the systematics of the excitation energy for the first $2^{+}$state which decreases smoothly between ${ }^{94} \mathrm{Kr}_{58}$ and ${ }^{96} \mathrm{Kr}_{60}$ [2]. The local character of the shape transition suggests that it is related to the interaction between specific proton and neutron orbitals. Finally, low-lying $0^{+}$states, indicating possible shape coexistence [3], have been identified in the $\mathrm{Zr}$ and $\mathrm{Sr}$ chains and, similar to the $2_{1}^{+}$state, an abrupt drop of the $0_{2}^{+}$energy is observed at $\mathrm{N}=60$. A shape coexistence scenario was proposed, where the $0_{2}^{+}$states for $\mathrm{N}<60$ correspond to a deformed configuration, which then becomes the ground state at $\mathrm{N}=60$, while the spherical configuration of the ground state for $\mathrm{N}<60$ becomes non-yrast.

Shape coexistence phenomena represent some of the most striking changes in nuclear structure observed in either particular atomic nuclei or between neighbours of an isotopic chain [3]. They are often associated with islands of inversion $[4,5]$, where spherical configurations, corresponding to doubly-closed shells, compete with deformed configurations. In the $\mathrm{Sr}$ and $\mathrm{Zr}$ isotopic chains, a spherical-to-deformed transition takes place when going from 58 to 60 neutrons, thus when the $v \mathrm{~g}_{7 / 2}$ orbital is being filled. Shell model calculations were performed for the $\mathrm{Zr}$ isotopic chain in an extended model space $[6,7]$ and propose a $\pi-v$ interaction between the spin-orbit partners $\pi 0 \mathrm{~g}_{9 / 2}$ and $v 0 \mathrm{~g}_{7 / 2}$ as the main mechanism for the shape change: as the $v\left(\mathrm{~g}_{7 / 2}, \mathrm{~h}_{11 / 2}\right)$ orbitals are being filled, the $\mathrm{Z}=40$ subshell gap between the $\pi 0 \mathrm{f}_{5 / 2}$ and $\pi 0 \mathrm{~g}_{9 / 2}$ effective single-particle energies (ESPE) is reduced, giving rise to multiple particle-hole excitations across the gap. In the calculations, the $0_{2}^{+}$states below $\mathrm{N}=60$ result from $2 \mathrm{p}-2 \mathrm{~h}$ proton excitations (with a possible $4 \mathrm{p}-4 \mathrm{~h}$ contribution) from the $p f$-shell into the $\pi\left(0 \mathrm{~g}_{9 / 2} 2 \mathrm{~d}_{5 / 2}\right)$ orbitals.

The structure of neutron-rich $\mathrm{Sr}$ isotopes has been studied extensively in the past, without a firm conclusion concerning shape coexistence in these nuclei. In ${ }^{96} \mathrm{Sr}$, the ground-state band was shown to have a vibrational-like character, and the small $B\left(E 2 ; 2_{1}^{+} \rightarrow 0_{1}^{+}\right)$value extracted from the lifetime of 7(4) ps [8] is consistent with a nearly spherical ground state. Two low-lying $0^{+}$states at 1229 and $1465 \mathrm{keV}$ were established [9] and interpreted as candidates for a deformed band head, supporting the shape coexistence scenario. An extremely strong electric monopole transition of $\rho^{2}(E 0)=0.185(50)$ was observed between these two states $[10,11]$, indicating the presence of a sizeable deformation and strong mixing between the configurations. In ${ }^{98} \mathrm{Sr}$, the ground-state band has a rotational character, and the large $\mathrm{B}(E 2)$ values between its members, deduced from lifetime measurements $[12,13,14,15,8,16,17]$, are consistent with a deformed character of the ground state. A low-lying $0_{2}^{+}$state at $215.3 \mathrm{keV}$ was established [18] and interpreted as the band head of a presumably spherical structure. A strong electric monopole transition of $\rho^{2}(E 0)=0.053(5)$ was measured between the $0_{2}^{+}$and the $0_{1}^{+}$states, again supporting the shape coexistence scenario $[18,19]$. 
We have recently reported on the spectroscopic quadrupole moments and reduced transition probabilities in ${ }^{96,98} \mathrm{Sr}$ measured by low-energy Coulomb excitation of post-accelerated radioactive ion beams at REX-ISOLDE, which provided firm evidence for shape coexistence and configuration inversion in the neutron-rich $\mathrm{Sr}$ isotopes [20, 21, 22]. In the present paper, we present a detailed comparison of the obtained deformation parameters with the available data in this mass region.

\section{Experimental details}

The experiments used post-accelerated radioactive beams of ${ }^{96} \mathrm{Sr}$ and ${ }^{98} \mathrm{Sr}$, which were delivered by the REX-ISOLDE facility at CERN with average intensities of $7 \times 10^{3}$ pps at $275.5 \mathrm{MeV}$ and $6 \times 10^{4}$ pps at $276.3 \mathrm{MeV}$, respectively, to the Coulomb excitation setup of the MINIBALL HPGe array [23]. Several different targets were used for this study in order to exploit the dependence of the Coulomb excitation cross section on the atomic numbers of the collision partners. De-excitation $\gamma$-ray spectra were sorted in prompt coincidence with scattered particles detected in the annular silicon strip detector. Doppler correction was applied on an event-by-event basis using both the position information from the particle detector and the electric segmentation of the MINIBALL detectors. The $\gamma$-ray spectra following Coulomb excitation of the ${ }^{96,98} \mathrm{Sr}$ beams are presented in [20, 22]. In ${ }^{96} \mathrm{Sr}$, the $2_{1}^{+} \rightarrow 0_{1}^{+}$transition was observed, together with excitation of the target nuclei: ${ }^{120} \mathrm{Sn}$ and ${ }^{109} \mathrm{Ag}$. For the ${ }^{120} \mathrm{Sn}$ target, where the statistics are larger, an additional weak transition corresponding to the $0_{2}^{+}$decay was present in the spectrum. In ${ }^{98} \mathrm{Sr}$, the rotational ground-state band was populated up to spin $6^{+}$and $8^{+}$using ${ }^{60} \mathrm{Ni}$ and ${ }^{208} \mathrm{~Pb}$ targets, respectively. The decay of the $2_{2}^{+}$state was also observed. The spectra presented in $[20,22]$ display some other gamma-ray lines that are not in coincidence with any known transition in ${ }^{98} \mathrm{Sr}$ or in ${ }^{98} \mathrm{Y}$. We attributed them to the beam contaminant ${ }^{98} \mathrm{Rb}$, for which no excited states have been firmly established before the present experiment[22]. This assumption is supported by the fact that similar low-energy $\gamma$ rays, in mutual coincidence, were identified in neighbouring nuclei.

The Coulomb excitation analysis was performed using the least squares fitting code GOSIA and its version capable of handling the mutual excitation of collision partners, GOSIA2 [24, 25, 26]. Both versions of the code use a standard $\chi^{2}$ function constructed from the measured $\gamma$-ray yields and those calculated from a set of electromagnetic matrix elements, both transitional and diagonal, between all states involved in the excitation process. Known spectroscopic data, such as lifetimes, mixing and branching ratios, can be used as additional data points in the minimisation procedure. The results are detailed in [20, 21, 22]. Based on the extracted $E 2$ matrix elements, the shape coexistence scenario in ${ }^{98} \mathrm{Sr}$ has been established: a highly deformed ground-state band coexists with a nearly spherical excited band built on the $0_{2}^{+}$state. The electromagnetic moments in the latter are similar to those in the ground state band in ${ }^{96} \mathrm{Sr}$, supporting the shape inversion at $\mathrm{N}=60$.

The diagonal E2 matrix elements extracted from the present Coulomb excitation analysis can be translated into spectroscopic quadrupole moments, defined in the laboratory frame, using the following formula:

$$
Q_{s}=\sqrt{\frac{16 \pi}{5}} \frac{1}{\sqrt{2 I+1}}(I, I, 2,0 \mid I, I)\langle I\|E 2\| I\rangle
$$




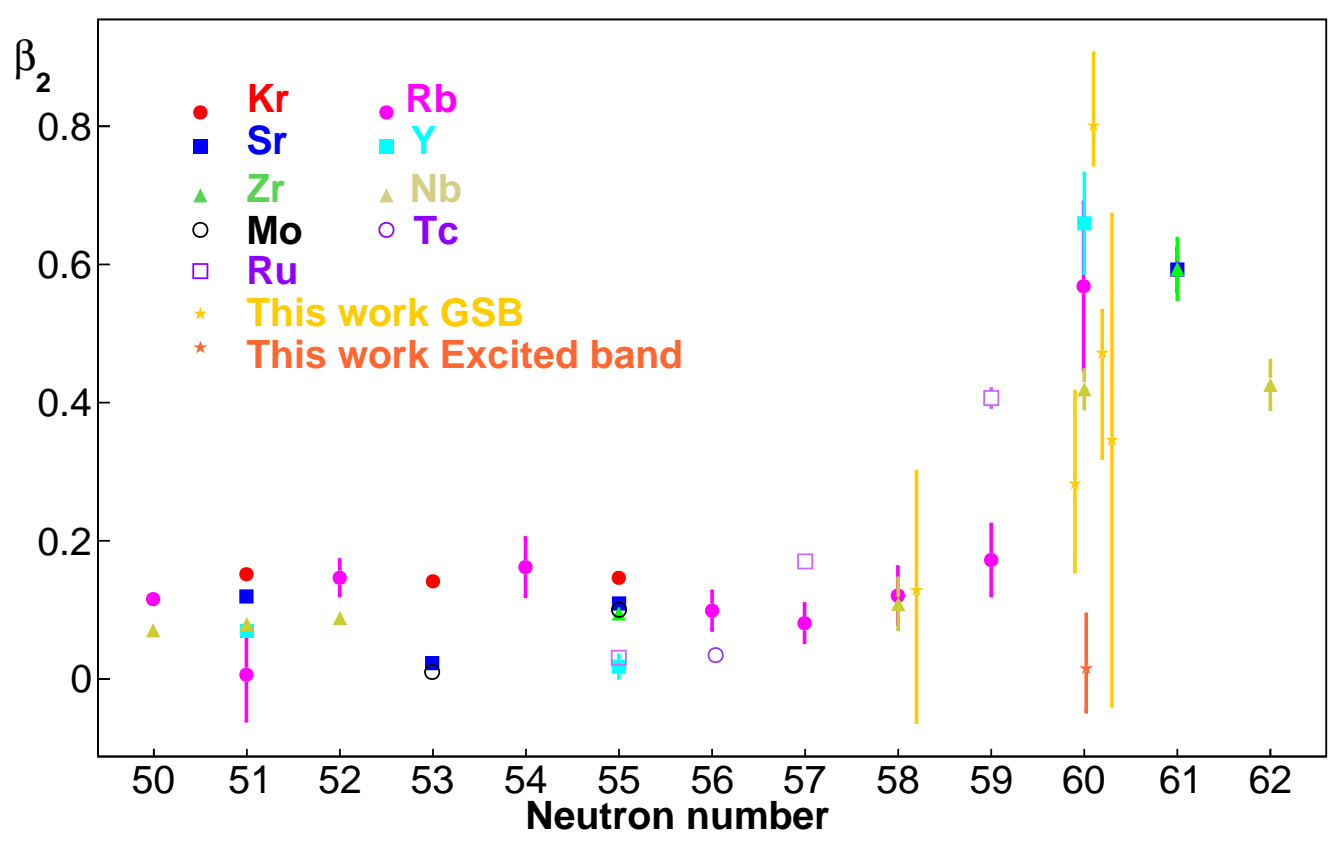

Figure 1: Quadrupole deformation parameter $\beta_{2}$ as a function of the neutron number in the $\mathrm{Kr}-\mathrm{Ru}$ isotopic chains. The values calculated from diagonal $E 2$ matrix elements measured in the present experiment for $\mathrm{J}>0$ states in ${ }^{96,98} \mathrm{Sr}$ are comared to those obtained from laser spectroscopy for the ground states of odd-mass nuclei [27].

Those can be compared to the spectroscopic quadrupole moments of the ground states obtained from laser spectroscopy for odd-mass neighbouring nuclei [27]. In order to eliminate mass and charge dependence, for the comparison we further convert them to quadrupole deformation parameters $\beta_{2}$ assuming axial symmetry:

$$
Q_{s}=\frac{3 \Omega^{2}-I(I+1)}{(I+1)(2 I+3)} Q_{0}=\frac{3 \Omega^{2}-I(I+1)}{(I+1)(2 I+3)} \frac{3}{\sqrt{5 \pi}} Z e R^{2} \beta_{2}
$$

where $\mathrm{R}$ is defined as $\mathrm{R}=1.25 \times A^{1 / 3}$. The comparison is shown in Fig. 1 as a function of the neutron number for existing data on odd-neutron and even-proton $\mathrm{Kr}, \mathrm{Sr}, \mathrm{Zr}$, Mo and Ru nuclei, and for even-neutron and odd-proton $\mathrm{Rb}, \mathrm{Y}, \mathrm{Nb}$ and $\mathrm{Tc}$ isotopes. Below $\mathrm{N}=60$, all these nuclei present a low deformation with $\beta_{2} \leq 0.2$. Our measurement of the spectroscopic quadrupole moment for the $2_{1}^{+}$state in ${ }^{96} \mathrm{Sr}$ fits well with the systematics. For $\mathrm{N} \geq 60$, the deformation of the ground state of odd-mass isotopes and in the ground state band of ${ }^{98} \mathrm{Sr}$ is consistently large, while the $\beta_{2}$ for the $2_{2}^{+}$in ${ }^{98} \mathrm{Sr}$ is compatible with the systematics for $\mathrm{N}<60$. This systematics can further completed by the spectroscopic quadrupole moments of the $2_{1}^{+}$states in ${ }^{94,96} \mathrm{Kr}[2,28]$ and ${ }^{98,100} \mathrm{Mo}$ [29, 30] extracted from low-energy Coulomb excitation experiments, leading to the picture presented in Fig2. The square size is proportional to $\beta_{2}$. The relevant proton and neutron orbitals in this valence space are also shown and illustrate, in the Quasi-SU3 and Pseudo-SU3 dynamical symmetries framework [31, 32], that the mechanism leading to the onset of deformation at $\mathrm{N}=60$ is similar to that explaining the well established islands of inversion [4, 5, 7].

The obtained $E 2$ matrix elements were further analysed using the quadrupole sum rules ap- 


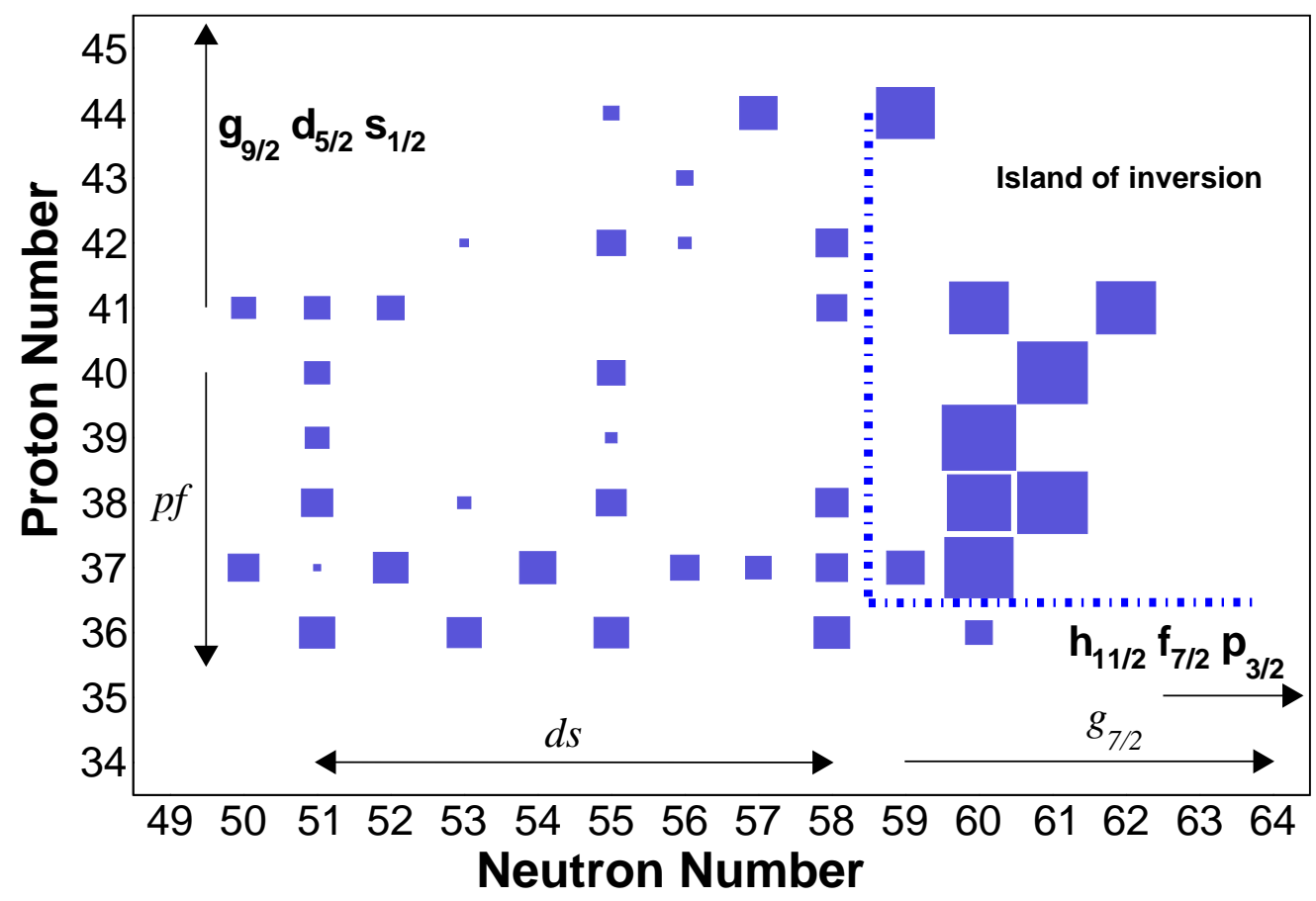

Figure 2: Experimental quadrupole deformation in the ground state for odd- $Z$ and/or odd-N nuclei and for the $2_{1}^{+}$state in even-even nuclei as a function of the proton and neutron number. The square size is proportional to $\beta_{2}$. The Pseudo (italic) and Quasi (bold) SU3 orbitals are shown.

proach [33,34]. This method relates experimentally determined $E 2$ matrix elements to deformation parameters, defined in the intrinsic frame of the nucleus, by constructing quadrupole invariants $\left\langle Q^{2}\right\rangle$ and $\left\langle Q^{3} \cos (3 \delta)\right\rangle$. The $Q$ parameter describes the magnitude of the deformation, while the $\delta$ parameter measures triaxiality. In order to determine the $\left\langle Q^{2}\right\rangle$ invariant for a $0^{+}$state, one has to measure absolute values of all $E 2$ matrix elements that couple the state in question with low-lying $2^{+}$states. To obtain the triaxiality parameter, $\langle\cos (3 \delta)\rangle$, more experimental information is needed, namely transitional matrix elements, together with their relative signs, between the state in question and $2^{+}$states, those coupling the $2^{+}$states one to another, as well as diagonal $E 2$ matrix elements of the $2^{+}$states. For ${ }^{96} \mathrm{Sr}$, the $\left\langle Q^{2}\right\rangle$ value for the ground state could be deduced from our measurement, corresponding to a rather low quadrupole deformation in agreement with the spectroscopic moment of the $2_{1}^{+}$state. In ${ }^{98} \mathrm{Sr}$, the $\left\langle Q^{2}\right\rangle$ values for the $0_{1,2}^{+}$states, obtained from the complete set of matrix elements, confirm an abrupt change in deformation between the ground state and the low-lying $0_{2}^{+}$state, consistent with the spectroscopic quadrupole moments measured for the $2_{1,2}^{+}$ states. The deformation of the ground state in ${ }^{96} \mathrm{Sr}$ and that of the low-lying $0_{2}^{+}$state in ${ }^{98} \mathrm{Sr}$ are similar, supporting again the scenario in which these two configurations interchange at $\mathrm{N}=60$. The triaxiality parameters $\langle\cos (3 \delta)\rangle$ for both $0_{1}^{+}$and $0_{2}^{+}$states in ${ }^{98} \mathrm{Sr}$, extracted from the present data, suggest a non-axially symmetric prolate shape. In view of the similarity between the $0_{2}^{+}$state in ${ }^{98} \mathrm{Sr}$ and the $0_{1}^{+}$state in ${ }^{96} \mathrm{Sr}$, one might also expect significant triaxiality for the ground state in ${ }^{96} \mathrm{Sr}$, which is consistent with the low negative value of the $2_{1}^{+}$spectroscopic quadrupole moment, reduced as compared to what would be expected for an axially symmetric shape. More information 
on the $2_{2,3}^{+}$states is clearly required to firmly establish the role of triaxiality in ${ }^{96} \mathrm{Sr}[20,21,22]$.

Triaxiality is often associated with the mixing of wave functions, hence we attempted to interpret the measured reduced matrix elements in ${ }^{98} \mathrm{Sr}$ using a simple two-state mixing model. In this model, the observed physical states $\left|I_{1}^{+}\right\rangle$and $\left|I_{2}^{+}\right\rangle$may be expressed as linear combinations of pure prolate and spherical configurations, $\left|I_{p}^{+}\right\rangle$and $\left|I_{s}^{+}\right\rangle$respectively:

$$
\begin{aligned}
& \left|I_{1}^{+}\right\rangle=+\cos \theta_{I} \times\left|I_{p}^{+}\right\rangle+\sin \theta_{I} \times\left|I_{s}^{+}\right\rangle \\
& \left|I_{2}^{+}\right\rangle=-\sin \theta_{I} \times\left|I_{p}^{+}\right\rangle+\cos \theta_{I} \times\left|I_{s}^{+}\right\rangle
\end{aligned}
$$

Experimental data, in particular $E 2$ matrix elements, can then be used to calculate the mixing amplitudes, $\cos ^{2} \theta_{I}$, between the two pure (unperturbed) configurations. Following the method described in our previous work [35], we have extracted from the complete set of $E 2$ matrix elements weak mixing angles for the $0^{+}$and $2^{+}$states wave functions [20, 21, 22].

The present data for ${ }^{98} \mathrm{Sr}$ show a sudden increase of the spectroscopic quadrupole moment at $\mathrm{J}^{\pi} \geq 4^{+}$that stabilises for higher spins [20, 21, 22]. In the shape coexistence framework, this effect could be explained by the mixing of the $2^{+}$wave functions that reduces the spectroscopic quadrupole moment of the $2_{1}^{+}$state. However, the two-state mixing model analysis indicates weak mixing. Hence, we tentatively attributed the observed reduction of the $\mathrm{Q}_{s}$ of the $2_{1}^{+}$state to a certain level of rigid triaxiality at low excitation energy.

We have performed beyond-mean-field calculations using the Gogny D1S interaction [36, 37] in a five-dimensional collective Hamiltonian $(5 \mathrm{DCH})$ formalism [38] for the ${ }^{96} \mathrm{Sr}$ and ${ }^{98} \mathrm{Sr}$ isotopes in order to obtain spectroscopic quadrupole moments and reduced transition probabilities for all states observed experimentally. The comparison of the calculated $\mathrm{B}(E 2)$ and $\mathrm{Q}_{s}$ with the experimental values is presented in Ref. [20,21, 22]. The theoretical calculation reproduces the onset of collectivity beyond $\mathrm{N}=60$, but its character is more gradual than the experimentally observed sharp rise at $\mathrm{N}=60$. The energy of the first excited $0^{+}$state is rather well reproduced, except for at $\mathrm{N}=60$ [20, 21, 22]. Finally, monopole transition strengths $\rho^{2}(E 0)$ in ${ }^{96,98} \mathrm{Sr}$ were calculated between the collective states obtained within the 5DCH approach up to spin 8 [22]. In ${ }^{96} \mathrm{Sr}$, large transition strengths are predicted between $\mathrm{O}_{1}^{+}, \mathrm{O}_{2}^{+}$and $\mathrm{O}_{3}^{+}$states. While the experimental $E 0$ strength for the $0_{3}^{+} \rightarrow 0_{2}^{+}$transition in ${ }^{96} \mathrm{Sr}$ ranks among the strongest known beyond $\mathrm{A}>40$, the $0_{2}^{+} \rightarrow 0_{1}^{+}$and $0_{3}^{+} \rightarrow 0_{1}^{+} E 0$ transitions have not been observed experimentally, which suggests on the contrary a very low $\rho^{2}(E 0)$. Significant $\rho^{2}(E 0)$ values, generally greater than $3 \cdot 10^{-2}$, were also calculated within the $5 \mathrm{DCH}$ approach for all transitions between $\mathrm{J}>0$ states in ${ }^{96} \mathrm{Sr}$, but there exist no experimental data to verify these predictions. In ${ }^{98} \mathrm{Sr}$, the experimental $\rho^{2}(E 0)$ value of $0.053(5)$ for the $0_{2}^{+} \rightarrow 0_{1}^{+}$transition can be compared to our present theoretical value of 0.178 . In addition, our calculations predict a $0_{3}^{+}$state coupled by strong $E 0$ transitions to both the ground state and the first excited $0^{+}$state. For spin $\mathrm{J}=2$, a very large $E 0$ strength is calculated between the rotational $2_{1}^{+}$ state and both the $2_{2}^{+} \gamma$-band head and the $2_{3}^{+}$state.

\section{Conclusions}

Shape coexistence in neutron-rich strontium isotopes at $\mathrm{N}=60$ was studied in low-energy Coulomb excitation experiments using radioactive ${ }^{96} \mathrm{Sr}$ and ${ }^{98} \mathrm{Sr}$ beams from the REX-ISOLDE 
facility, CERN. Reduced transition probabilities and spectroscopic quadrupole moments for lowlying states in ${ }^{96,98} \mathrm{Sr}$ were extracted from the measured $\gamma$-ray yields. In ${ }^{96} \mathrm{Sr}$, the spectroscopic quadrupole moment of the first $2^{+}$state was found to be small and negative, corresponding to a weak prolate deformation. In ${ }^{98} \mathrm{Sr}$, the large and negative spectroscopic quadrupole moments in the ground state band demonstrate its prolate rotational character, while the value close to zero obtained for the $2_{2}^{+}$state confirms the shape coexistence between highly-deformed prolate and spherical configurations in this nucleus. The comparison of the $\mathrm{B}(E 2)$ values and the spectroscopic quadrupole moments between the $2_{1}^{+}$state in ${ }^{96} \mathrm{Sr}$ and the $2_{2}^{+}$state in ${ }^{98} \mathrm{Sr}$ underlines their similarity and further supports the shape inversion when crossing the $\mathrm{N}=60$ line. This conclusion is even more evident when the experimental information on quadrupole deformation in ${ }^{96,98} \mathrm{Sr}$ is presented in context of other measurements of spectroscopic quadrupole moments in this mass region. The reduced E2 matrix elements were interpreted in a phenomenological two band mixing model. The results support the weak mixing scenario between prolate and spherical configurations in the wave functions of the $0^{+}$states in ${ }^{98} \mathrm{Sr}$, in spite of their proximity in energy. The increase of the spectroscopic quadrupole moments observed beyond the $2_{1}^{+}$state can be attributed to a certain level of triaxiality in the ground state. This interpretation is further supported by the results of the quadrupole sum rules method applied to the $0^{+}$states in ${ }^{98} \mathrm{Sr}$, where the values of the quadrupole invariant $\left\langle\mathscr{Q}^{3} \cos (3 \delta)\right\rangle$ for both states suggest a significant degree of triaxiality. The experimental results were compared to 5DCH calculations using the Gogny D1S interaction, which reproduce the shape coexistence between deformed and spherical configurations in ${ }^{96} \mathrm{Sr}$ and ${ }^{98} \mathrm{Sr}$ and the change of the ground state deformation at $\mathrm{N}=60[20,21,22]$.

\section{References}

[1] S. Naimi et al. Phys. Rev. Lett., 105:032502, Jul 2010.

[2] M. Albers et al. Phys. Rev. Lett., 108:062701, Feb 2012.

[3] Kris Heyde and John L. Wood. Rev. Mod. Phys., 83:1467-1521, Nov 2011.

[4] E. Caurier, G. Martínez-Pinedo, F. Nowacki, A. Poves, and A. P. Zuker. Rev. Mod. Phys., 77:427-488, Jun 2005.

[5] B.A. Brown. Progress in Particle and Nuclear Physics, 47(2):517 - 599, 2001.

[6] K. Sieja, F. Nowacki, K. Langanke, and G. Martinez-Pinedo. Phys. Rev. C, 79:064310, Jun 2009.

[7] Tomoaki Togashi, Yusuke Tsunoda, Takaharu Otsuka, and Noritaka Shimizu. Phys. Rev. Lett., 117:172502, Oct 2016.

[8] H. Mach et al. Nucl. Phys. A, 523(2):197 - 227, 1991.

[9] G. Jung et al. Phys. Rev. C, 22:252-263, Jul 1980.

[10] K. Kawade et al. Zeitschrift für Physik A Atoms and Nuclei, 304(4):293-299, 1982.

[11] G. Lhersonneau et al. Phys. Rev. C, 49:1379-1390, Mar 1994.

[12] H. Mach et al. Phy. Lett. B, 230:21 - 26, 1989.

[13] H. Ohm et al. Z.Phys., A327:483, 1987.

[14] H. Mach et al. Nucl. Inst. and Meth. A, 280(1):49 - 72, 1989. 
[15] G. Lhersonneau et al. Phys. Rev. C, 65:024318, Jan 2002.

[16] A. G. Smith et al. Phys. Rev. Lett., 77:1711-1714, Aug 1996.

[17] A. G. Smith et al. Phys. Rev. C, 86:014321, Jul 2012.

[18] F. Schussler et al. Nucl. Phys. A, 339(3):415 - 428, 1980.

[19] J. Park et al. Phys. Rev. C, 93:014315, Jan 2016.

[20] E. Clément et al. Phys. Rev. Lett., 116:022701, Jan 2016.

[21] E. Clément et al. Phys. Rev. Lett., 117:099902, Aug 2016.

[22] E. Clément et al. Phys. Rev. C, 94:054326, Nov 2016.

[23] N. Warr et al. The miniball spectrometer. Eur. Phys. J A, 49(3):1-32, 2013.

[24] T. Czosnyka, D. Cline, and C. Y. Wu. Bull. Am. Phys. Soc., 28:745, 1983.

[25] GOSIA User's Manual, page http://www.slcj.uw.edu.pl/gosia/.

[26] M. Zielińska et al. The European Physical Journal A, 52(4):99, 2016.

[27] N.J. Stone. Atomic Data and Nuclear Data Tables, 1112̆013112:1 - 28, 2016.

[28] M. Albers et al. Nucl. Phys. A, 899(0):1 - 28, 2013.

[29] M. Zielińska et al. Nuclear Physics A, 712(1):3 - 13, 2002.

[30] K. Wrzosek-Lipska et al. Phys. Rev. C, 86:064305, Dec 2012.

[31] A. P. Zuker, J. Retamosa, A. Poves, and E. Caurier. Phys. Rev. C, 52:R1741-R1745, Oct 1995.

[32] A. P. Zuker, A. Poves, F. Nowacki, and S. M. Lenzi. Phys. Rev. C, 92:024320, Aug 2015.

[33] Douglas Cline. Ann. Rev. of Nucl. and Part. Sc., 36:683-716, 1986.

[34] J. Srebrny et al. Int. J. Mod. Phys. E, 20:422, 2011.

[35] E. Clément et al. Phys. Rev. C, 75:054313, May 2007.

[36] J. Dechargé and D. Gogny. Phys. Rev. C, 21:1568-1593, Apr 1980.

[37] J.F. Berger et al. Comp. Phys. Com., 63(1-3):365 - 374, 1991.

[38] J. P. Delaroche et al. Phys. Rev. C, 81:014303, Jan 2010. 